\title{
AGREGANDO VALOR A LAS FRUTAS DEL CERRADO BRASILEÑO: ENCAPSULACIÓN DE ACEITE DE PEQUI A TRAVÉS DEL SECADO POR ATOMIZACIÓN
}

\author{
Joyce Maria Gomes da Costa ${ }^{1}$, Ariel Antonio Campos Toledo Hijo ${ }^{2}$, Eric Keven Silva ${ }^{3}$, Soraia Vilela Borges ${ }^{4}$, \\ Gerson Reginaldo Marques ${ }^{5}$
}

\begin{abstract}
RESUMEN
El aceite de pequi es un producto rico nutricionalmente y su encapsulación por spray drying tiene como finalidad aumentar su vida útil y facilidad de utilización. Este trabajo tiene como objetivos la caracterización física, química y morfológica del aceite de pequi encapsulado por spray drying. Fue utilizado una emulsión $10 \%(\mathrm{p} / \mathrm{p})$ teniendo como encapsulantes una mezcla de 25/50/25 de respectivamente, goma arábica, almidón modificado y maltodextrina. Las condiciones de secado fueron: 180 y $105{ }^{\circ} \mathrm{C}$ del aire de entrada y salida del secador, respectivamente, y sistema de atomización en pico doble fluido, con flujo de alimentación de $0.96 \mathrm{~L}^{\mathrm{h}} \mathrm{h}^{-1}$. El proceso utilizado resulta en un rendimiento de $36 \%$ de aceite encapsulado, partículas esféricas, lisas y algunas con invaginaciones, alta polidispersidad en cuanto al tamaño y una tendencia a aglomeraciones de las partículas. El producto presentó buena solubilidad (79.38\%, aproximado), baja humectabilidad (954s, aproximado), y luminosidad se aproxima al blanco y tiende al rojo y amarillo.
\end{abstract}

Palabras-clave: Caryocar brasiliense, nebulización, carotenoide, encapsulación, spray drying

\section{ABSTRACT \\ ADDING VALUE TO THE FRUITS OF THE BRAZILIAN CERRADO: ENCAPSULATION OF PEQUI OIL BY SPRAY DRYER}

The pequi oil is a nutritionally rich product and the purpose of the encapsulation using spray drying is extending it shelf life and making it easier to handle. The aim of this work was to characterize physically, chemically and morphologically the pequi oil microparticles produced by spray drying. It was used an emulsion $10 \%$ (w/w) and a wall system mixture of 25/50/25 consisted by gum arabic, modified starch and maltodextrin, respectively. The drying conditions were: 180 and $105^{\circ} \mathrm{C}$ as inlet and outlet air temperature, respectively, and a double peak fluid atomization system with feed rate at $0.96 \mathrm{~L} \cdot \mathrm{h}^{-1}$. The results of the process show a $36 \%$ of microencapsulation efficiency, spherical particles, smooth, and invaginations, high polydispersity as a function of size and a tendency of agglomerations of the particles. The product shows a great solubility (approximately 79.38\%), a low wettability (approximately 954s), and it luminosity is close to the white and tends to red and yellow.

Keywords: Caryocar brasiliense, nebulization, carotenoid, encapsulation, spray drying

Recebido para publicação em 16/07/2015. Aprovado em 16/06/2016.

1 - Ingeniera de alimentos, profesor da UFVJM/ICT/Diamantina-MG, joyce.costa@ict.ufvjm.edu.br

2 - Ingeniero de alimentos, universitario de doctorado da UNICAMP, arielhi@fea.unicamp.br

3 - Ingeniero de alimentos, universitario de doctorado da UNICAMP, erickeven@hotmail.com

4 - Ingeniera química, profesor da UFLA/DCA/Lavras-MG, sborges@dca.ufla.br

5 - Ingeniero químico, universitario de doctorado da UFLA/DCA/Lavras-MG, greginaldo@gmail.com 


\section{INTRODUCCIÓN}

El pequicero (Caryocar brasiliese Camb.) es una planta típica de la cierra brasileña cuyos frutos son muy ricos en aceites, los cuales presentan en su composición ácidos grasos insaturados y carotenoides, teniendo muchas aplicaciones en los sectores alimenticio, farmacéutico y cosmético (AQUINO y col., 2007). Entre estas aplicaciones se incluyen la prevención de algunos tipos de cáncer, en la inhibición de las mucosas contra úlceras gástricas, en la capacidad de prevenir la fotosensibilización en ciertas enfermedades de la piel, en el aumento de la respuesta inmunológica a determinados tipos de infección y en las propiedades de anti-envejecimiento, reducción del colesterol total y de la presión arterial, además de presentar actividad antioxidante en grasas y aceites y ser fuente de vitamina A (MIRANDA-VILELAy col., 2009). Además de eso, el aceite es aromático, siendo muy utilizado en la culinaria brasileña (MAIA, ANDRADE \& SILVA, 2008).

Sin embargo, la exposición de productos lipídicos al calor, oxígeno, luz, aire y humedad puede causar cambios estructurales, reduciendo de esta forma su actividad como vitamina y anti oxidante (O'BRIEN, 2004).

Ante estos fenómenos, se ha estudiado diversos mecanismos de protección de compuestos sensibles a oxidación o volatilización, siendo la microencapsulación por secado por atomización uno de los métodos más utilizados para este fin debido a la rápida exposición del material a temperaturas menores que $80{ }^{\circ} \mathrm{C}$, además de la alta producción y bajo costo en relación a otros métodos de conservación (TEIXEIRA y col., 2004; BARANAUSKIENÉ $y$ col., 2006; CHAMBI $y$ col., 2008). Esta técnica está basada en la captura del compuesto activo por una pared compuesta de aditivos que tengan capacidad de emulsionar la solución, aumentar la temperatura de transición vítrea de la solución para evitar un colapso durante el secado y formar película resistente para su posterior secado (MADENE y col.,2006; GHARSALLAOUOI $y$ col., 2007).

El objetivo de este trabajo consiste en caracterizar las propiedades químicas, físicas y morfológicas de muestras de micropartículas de aceite de pequi obtenidas por secado por atomización.

\section{MATERIAL Y MÉTODOS}

El aceite de pequi fue adquirido en el comercio local, la maltodextrina GLOBE® 1905 y DE 20 y Capsul Snow Flake ${ }^{\circledR}$ E6131 (almidón y maíz químicamente modificado), fueron proveídos por la Corn Products, Mogi Guaçu, SP, Brasil. La goma arábica fue adquirida en RM-MAIA (Belo Horizonte-MG, Brasil).

La emulsión $(10 \% \mathrm{p} / \mathrm{p})$ fue preparada con aceite de pequi (10\% de los sólidos) y material de pared (90\% de los sólidos) compuesto de 25 $\%$ de goma arábica, $50 \%$ de almidón modificado y $25 \%$ de maltodextrina, de acuerdo con pruebas preliminares de desempeño del secador spray drying. Inicialmente, la maltodextrina y la goma arábica fueron diluidos en agua deionizada a 60-70 ${ }^{\circ} \mathrm{C}$, usando el homogenizador Ultraturrax en una velocidad de $20.000 \mathrm{rpm}$ (revoluciones por minuto) durante 30 minutos. En seguida, se adicionó el Capsul a $82{ }^{\circ} \mathrm{C}$ manteniendo la homogenización. Después de la dilución completa de los materiales de pared, fue adicionado el aceite de pequi hasta obtener una emulsión completamente homogénea en una rotación de $20.000 \mathrm{rpm}$ durante 20 minutos.

La emulsión formada fue sometida al secado a través de un secador por atomización, de banco, de la marca LAMBAQ do Brasil, modelo MSD 1.0 (Ribeirão Preto, SP-Brasil). Las condiciones de operación del secador fueron: $180 \pm 2$ y $105 \pm 2{ }^{\circ} \mathrm{C}$ del aire de secado de entrada y salida del secador, respectivamente, y sistema de atomización en pico doble fluido con apertura de $1.2 \times 10^{-3} \mathrm{~m}$, flujo de alimentación de $2.97 \times 10^{-7} \mathrm{~m}^{3} \mathrm{~s}^{-1}$, y flujo de aire de entrada de $5.8 \times 10^{-4} \mathrm{~m}^{3} \mathrm{~s}^{-1}$ (COSTA y col. 2013).

Los análisis químicos de las micropartículas de aceite de pequi fueron realizadas en triplicado. Los métodos utilizados siguieron las normas de la AOAC, 2000. La determinación del contenido de humedad fue realizada por método gravimétrico, a temperatura de $105^{\circ} \mathrm{C}$, hasta peso constante. El extracto etéreo fue obtenido por extracción con éter etílico, por 5 horas, usando el aparato tipo Soxhlet TECNAL (Piracicaba, SP, Brasil). El contenido de nitrógeno fue determinado por el método de Micro-

\section{REVENG}


Kjeldahl comprendiendo las etapas de digestión con $\mathrm{H}_{2} \mathrm{SO}_{4}$ (ácido sulfúrico), destilación con solución de $\mathrm{NaOH}$ (hidróxido de sódio) $50 \%$ y titulación con solución de $\mathrm{HCl}$ (ácido clorídrico) $0,02 \mathrm{~N}$, utilizando el factor de conversión para proteína bruta equivalente a 6.25 . El contenido de cenizas fue determinado por el método gravimétrico con incineración en mufla a $550{ }^{\circ} \mathrm{C}$.

La actividad de agua (Aw) de las muestras fueron obtenidas por lectura directa en termohigrómetro AQUA-LAB digital, modelo CX-2 (Decagon Devices Inc., EUA), con temperatura controlada de $25.0 \pm 0.5^{\circ} \mathrm{C}$.

El color de las micropartículas de aceite de pequi procedió conforme los valores $\mathrm{L}^{*}$ (parámetro del color brillo), a* (parámetro del color $a^{*}$ ), $\mathrm{b}^{*}$ (parámetro del color $\mathrm{b}^{*}$ ), donde fueron determinados con el aparato colorímetro Minolta modelo CR 400, trabajando con $\mathrm{D}_{65}$ (luz del día) y usando los padrones CIELab: en que $\mathrm{L}^{*}$ varia de 0 (negro) a 100 (blanco), a* varia de verde (-) al rojo $(+), b^{*}$ varia de azul (-) al amarillo $(+)$.

La humectabilidad fue determinada por el método HLA (1999).

La solubilidad fue calculada por el método descrito por Eastman \& Moore (1984), modificado por Cano-Chauca y col. (2005).

Las muestras fueron preparadas de acuerdo con Alves (2004). Las micropartículas de aceite de pequi fueron montadas en suportes de aluminio (stubs) y cubiertos con oro (evaporador Balzers SCD 050) para observación en microscopio electrónico de barrido LEO EVO 40. Las imágenes fueron generadas y registradas digitalmente, con obtención de diversas imágenes para cada muestra en las condiciones de trabajo de $20 \mathrm{kV}$ y distancia de trabajo de $10.5 \mathrm{~mm}$. Las electromicrografías generadas fueron grabadas y abiertas en el Software Photopaint del paquete Corel Draw 9.

Los diámetros medios de las micropartículas fueron determinadas por dispersión de luz, utilizando laser de alta potencia en un equipamiento Mastersizer 2000. La dispersión de las partículas fue realizada en la unidad de dispersión del equipamiento, utilizando $600 \mathrm{~mL}$ de isopropanol PA (Synth) como medio dispersante. El tiempo de agitación de la solución fue de 15 minutos, y fueron efectuadas 15 medidas con intervalo de
10 segundos. Los resultados fueron analizados a través de la teoría de Fraunhofer, donde el índice de refracción de la partícula fue desconsiderado, y el modelo de análisis fue de partícula esférica. El ultra-son fue usado por 4 minutos.

\section{RESULTADOS Y DISCUSIÓN}

Conforme se observa en la Cuadro 1 el contenido de humedad de la muestra es muy bajo, típico de productos deshidratados por este proceso, lo que limita el crecimiento microbiano y contribuye para la estabilidad del producto. El extracto etéreo corresponde a la fracción lipídica de la muestra y, de esta forma, se puede estimar que la eficiencia de encapsulación del aceite de pequi fue de $33.5 \%$, considerada baja en relación a otros trabajos (BARANAUSKIENÉ, 2006; TURCHIULI y col., 2006) cuyos valores están en torno de 50-80 \%, lo que llevará a la optimización del proceso en otras condiciones de secado. En relación a la proteína y cenizas se observa valores inferiores a los de la almendra (27.7 e $4.39 \%$ b.s, respectivamente) (LIMA $y$ col., 2007) debido al proceso de extracción y purificación del aceite. Los carbohidratos corresponden a los aditivos adicionados.

Las micropartículas de aceite de pequi producidas por atomización presentaron valores de actividad superiores a los encontrados por Turchiuli y col. (2006), para encapsulación de aceite vegetal (Cuadro 2). La actividad de agua está relacionada, juntamente con otros factores, como temperatura y exposición a la luz y oxígeno, a la estabilidad y al tiempo de almacenamiento, (vida útil) de alimentos, humedad relativa o la presión de vapor del aire. En general, valores inferiores a 0.6 inhiben diversas reacciones indeseables como oxidación, reacción de Maillard, acción de enzimas y desarrollo de microorganismos (FENNEMA, 1996).

Por el análisis de colorimetría fueron encontrados los valores $81.62,4.71$ y 12.035 , para $\mathrm{L}^{*}, \mathrm{a}^{*} \mathrm{y} \mathrm{b}^{*}$ respectivamente. Lo que indica que la luminosidad de las micropartículas conteniendo aceite de pequi se aproxima al blanco y tiende al rojo y amarillo.

El color instrumental del aceite tiene una estrecha correlación con los pigmentos existentes conforme 
Cuadro 1. Composición centesimal de las micropartículas de aceite de pequi centesimal composition of the pequi oil microparticles

\begin{tabular}{cc}
\hline Constituyente & Valores $(\mathrm{g} / 100 \mathrm{~g})$ \\
\hline Humedad & $3.46 \pm 0.46$ \\
Extracto Etéreo & $3.35 \pm 0.1$ \\
Proteína & $5.15 \pm 0.09$ \\
Ceniza & $0.93 \pm 0.1$ \\
Fibra & 0.000 \\
Carbohidratos* & 87.11 \\
\hline
\end{tabular}

* determinado por diferencia

Cuadro 2. Propiedades físicas y químicas del aceite de pequi encapsulado chemical an physical properties of the encapulated oil pequi

\begin{tabular}{cc}
\hline Propiedad & Valor \\
\hline Actividad de agua & $0.50 \pm 0.05$ \\
Solubilidad & $79.38 \pm 1.47$ \\
Humectabilidad & $954 \mathrm{~s} \pm 66.09$ \\
$\mathrm{~L}^{*}$ & $81.62 \pm 4.62$ \\
$\mathrm{a}^{*}$ & $4.71 \pm 0.30$ \\
$\mathrm{~b}^{*}$ & $12.04 \pm 0.35$ \\
\hline
\end{tabular}

extensiva investigación relatada por Moyano y col., (2008) y en el caso el tono es amarillado debido a los carotenoides presentes (AZEVEDO-MELEIRO \& RODRIGUEZ-AMAYA, 2004; AQUINO y col., 2007). El secado o almacenado tiende a oxidar este pigmento y oscurecer el producto (ARABHOSSEINI y col., 2007).

Las micropartículas de aceite de pequi se presentaron bastante solubles en relación al extracto de tomate deshidratado $(22.27 \mathrm{~g} / 100 \mathrm{~g})$ (SOUSA $y$ col., 2008) y presentaron una humectabilidad mejor que la obtenida por Turchiuli y col., (2006) (28 minutos) para aceite vegetal encapsulado, y para extracto de tomate deshidratado (19.5 minutos) (SOUSA y col., 2008).

Se verifica en la Figura 1A que parte de las micropartículas conteniendo aceite de pequi, producidas a través del secado por atomización, presentan paredes continuas, arredondeadas, sin poros aparentes, fisuras, rajaduras o rompimientos. La integridad de la pared es importante para garantizar una mejor retención del relleno y menor permeabilidad al oxígeno y vapor de agua. Se observó todavía la presencia de concavidades y aplastamientos en la superficie. Esas imperfecciones de la superficie de las partículas ya fueron constatadas por otros autores y son características del proceso de secado por atomización, pudiendo estar asociadas a las características del agente encapsulante y también a las condiciones de proceso (ASCHERI, 1999; TRINDADE \& GROSSO, 2000; BERTOLINI, SIANI \& GROSSO, 2001).

La Figuras 1B-C presenta la variación de tamaño (polidispersidad) de las micropartículas. La alta polidispersidad es una característica de partículas obtenidas por atomización, pues durante la pulverización, las gotas formadas no tienen tamaños uniformes. Procesos como spray drying, spray cooling, gelificación iónica, que pueden utilizar atomizadores en formatación de las partículas, tienden a producir muestras con alta polidispersidad. 

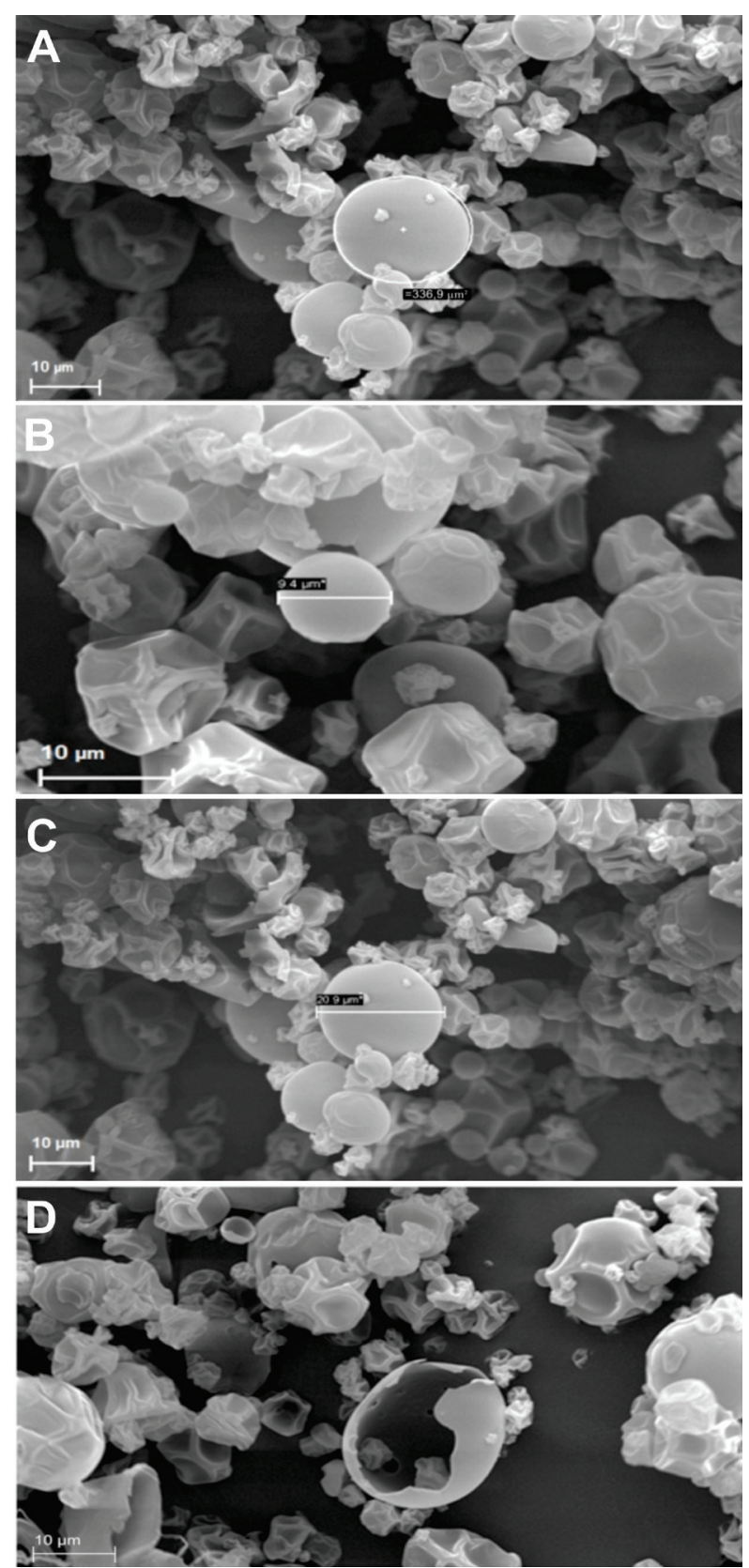

Figura 1. Análisis morfológica de las micropartículas de aceite de pequi morphological analysis of microparticles of oil pequi.

Se observó una tendencia a formación de aglomerados, lo que también es relativamente común en polvos producidos a través de la atomización (BHANDARI y col., 1992; CHAMBI y col., 2008). Según Trindade \& Grosso (2000), aglomerados pueden contener material de relleno en sus intersticios, lo que puede auxiliar en la retención de estos; todavía, según Colombo \&
Gerber, (1991), este fenómeno es importante porque confiere mayor protección al relleno.

Algunas micropartículas cuando se quiebran demuestran poseer pared porosa (Figura 1D). De acuerdo con Sheu \& Rosenberg (1998) y Teixeira y col., (2004), muestras producidas en spray drying poseen, generalmente, un formato esférico y son huecas, siendo que la formación de la vacuola se origina de un proceso de encogimiento que ocurre después de la solidificación de la pared seguida por la expansión de las burbujas de aire retenidas dentro de la gota. Los mecanismos asociados con la formación de este espacio vacío en el centro están relacionados a la expansión de las cápsulas durante las últimas etapas del proceso del secado. La presencia de invaginaciones es probablemente debida al encogimiento de las gotas durante las etapas iniciales del proceso del secado (SHEU \& ROSENBERG, 1998). Los mismos autores citan todavía que la formación de la vacuola sea causada por la microencapsulación por atomización utilizando goma arábica como agente encapsulante. Luego el material encapsulado queda retenido en la pared de estas y no en el centro. En la Figura 1D es posible verificar la presencia de vacuolas en toda la extensión de la pared, donde, probablemente, contenía el aceite de pequi.

La Cuadro 3 presenta los diámetros de las micropartículas fornecidos a través del Mastersizer (2000), y la Figura 2 muestra la distribución de tamaño de las micropartículas representadas a través de D (4.3) que corresponde al diámetro medio volumétrico. Este diámetro corresponde al diámetro de las partículas esféricas que poseen el mismo volumen medio de las partículas constituyentes del sistema.

A través del histograma presentado en la Figura 2 , se puede observar que hubo un comportamiento bimodal, el cual es particularmente interesante tomando en consideración el almacenamiento del polvo, una vez que la población de partículas menores puede penetrar en los espacios presentes entre las partículas mayores, ocupando un espacio menor. 
Cuadro 3. Diámetros de las micropartículas de aceite de pequi diameter of the pequi oil microparticles.

\begin{tabular}{lllll}
\hline $\begin{array}{l}\mathrm{D}_{(0.1)} \\
\mu \mathrm{m}\end{array}$ & $\begin{array}{l}\mathrm{D}_{(0.5)} \\
\mu \mathrm{m}\end{array}$ & $\begin{array}{l}\mathrm{D}_{(0.9)} \\
\mu \mathrm{m}\end{array}$ & $\begin{array}{l}\mathrm{D}_{(3.2)} \\
\mu \mathrm{m}\end{array}$ & $\begin{array}{l}\mathrm{D}_{(4.3)} \\
\mu \mathrm{m}\end{array}$ \\
\hline 2.563 & 11.185 & 23.860 & 5.554 & 12.412 \\
\hline
\end{tabular}

$\mathrm{D}(4.3 \mu \mathrm{m})$ : diámetro medio en volumen

$\mathrm{D}(0.1 \mu \mathrm{m})$ : diámetro de partícula correspondiente a $10 \%$ de la distribución acumulada

$\mathrm{D}(0.5 \mu \mathrm{m})$ : diámetro de partícula correspondiente a $50 \%$ da distribución acumulada

$\mathrm{D}(0.9 \mu \mathrm{m})$ : diámetro de partícula correspondiente a $90 \%$ da distribución acumulada

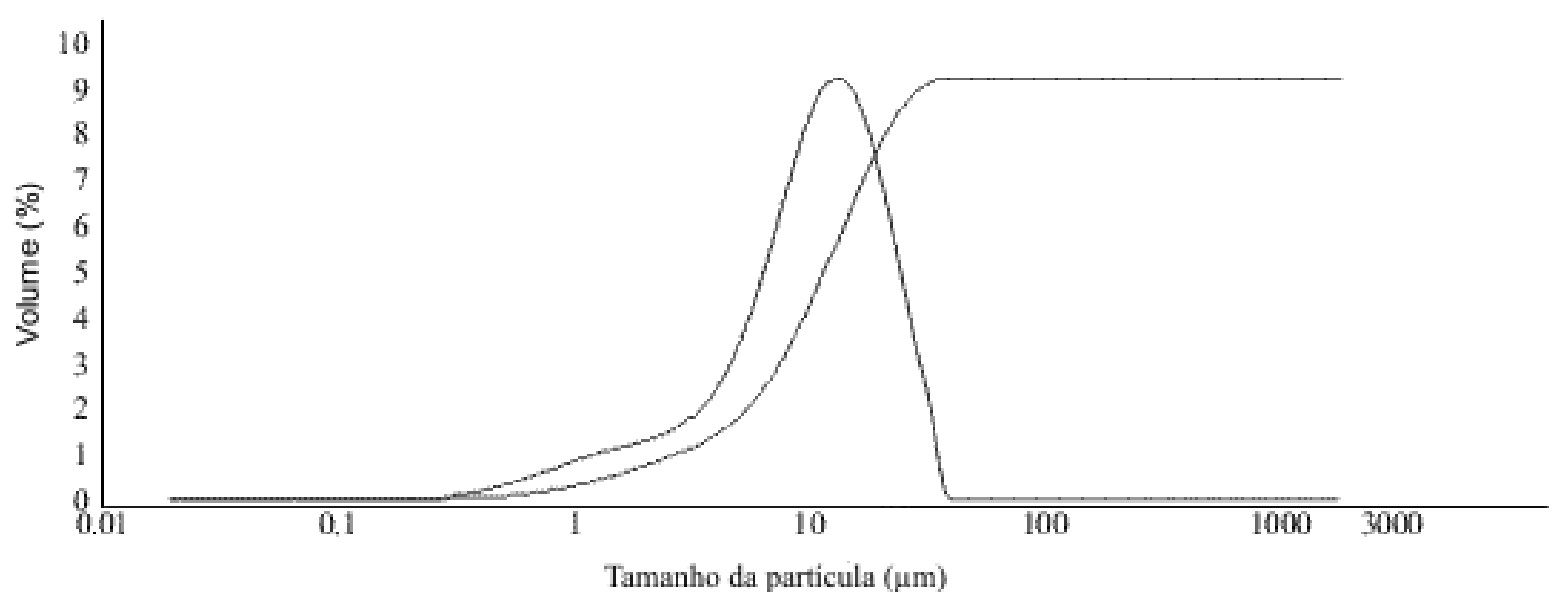

Figura 2. Distribución de tamaño de las micropartículas size distribuition of pequi oil microparticles.

Cerca de $9.13 \%$ de las partículas presentaron tamaño entre, aproximadamente, 13.18 y $17.9 \mu \mathrm{m}$ y partículas mayores que $50 \mu \mathrm{m}$ y menores que $0.3 \mu \mathrm{m}$ obtuvieron porcentajes mínimos. Chambi y col. (2008), encapsularon compuestos lipídicos utilizando glucosa, caseína e caseína hidrolizada, y se obtuvo diámetro medio de las partículas entre 12.9 y $21.7 \mu \mathrm{m}$, siendo semejantes a la variación obtenida en este trabajo.

Según Benet \& Hoener (2000), partículas pequeñas poseen una mayor velocidad de disolución debido al aumento del área superficial. Luego, como las micropartículas de aceite de pequi presentaron diámetros medios próximos a $13 \mu \mathrm{m}$, estas tienden a presentar una buena dispersión granulométrica y presentaron tamaño característico de productos procesados en spray dryer (BUFFO \& REINECCIUS, 2002; ONEDA \& RÉ, 2003; RODRÍGUEZ-HUEZO y col., 2004).

\section{CONCLUSIONES}

- El proceso utilizado resultó en un rendimiento de $36 \%$ de aceite encapsulado, partículas esféricas, lisas y algunas con invaginaciones, y alta polidispersidad en cuanto al tamaño y una tendencia de aglomeración de las partículas.

- El producto presentó buena solubilidad, baja humectabilidad, y su luminosidad se aproxima al blanco y tiende al rojo y amarillo.

\section{AGRADECIMIENTOS}

Queremos expresar nuestro agradecimiento a la FAPEMIG por el aporte financiero y al CNPq por las becas de doctorado e iniciación científica.

\section{REFERENCIAS BIBLIOGRÁFICAS}

ALVES, E. Introdução à microscopia eletrônica de varredrura e de transmissão. Editorial FAEPE, Universidade Federal de Lavras, Brasil, 2004, 43p.

AQUINO, L.P.; BORGES, S.V.; QUEIROZ, F.; ANTONIASSI, R.; CIRILLO, M.A. Extraction of

\section{REVENG}


oil from pequi fruit (Caryocar brasiliense Camb.) using several solvents and their mixtures, Grasas y Aceites, v.62, p.245-252, 2007.

ASCHERI, D.P.R. Estudo das características de adsorção de água e da estabilidade das microcápsulas de óleo essencial de laranja na seleção de material de parede, Ciência e Tecnologia de Alimentos, v.19, p.367-373, 1999.

ARABHOSSEINI, A.; HUISMAN, W.; VAN BOXTEL A.; MÜLLE, J. Long-term effects of drying conditions on the essential oil and color of tarragon leaves during storage, Journal of Food Engineering, v.79, p.561-566, 2007.

AOAC (2000). Official methods of analysis. Gaithersburg. Association of Official Analytical Chemists. $17^{\text {a }}$ Ed.

AZEVEDO-MELEIRO, C.H.; RODRIGUEZAMAYA, D.B. Confirmation of the identify of the carotenoids of tropical fruits by HPLC-DAD and HPLC-MS. Journal of Food Composition and Analysis, v.17, n.3-4, p.385-396, 2004.

BARANAUSKIENÉ, R.; VENSKUTONIS, P.R.; DEWETTINCK, K.; VERHÉ, R. Properties of oregano (Origanum vulgare L.), citronella (Cymbopogon nardus G.) and marjoram (Majorana hortensis L.) flavors encapsulated into mil proteinbased matrices, Food Research International, v.39, p.413-425, 2006.

BENET, L.Z.; HOENER, B. Factors influencing drug absorption and drug availability. In: Modern Pharmaceutics, (GSBanker \& CT Rhodes, Ed.), p.93-117. Marcel Dekker Inc, New York, 2000.

BERTOLINI, A.C.; SIANI, A.C.; GROSSO, C.R.F. Stability of monoterpenes of encapsulated in gum arabic by spray drying, Journal Agriculture and Food Chemistry, v.49, p.780-785, 2001.

BHANDARI, B.R.; DUMOULIN, E.D.; RICHARD, H.M.J.; NOLEAU, I.; LEBERT, A.M. Flavor encapsulation by spray drying: application to citral and linalyl acetate. Journal of Food
Science, Chicago, v.57, n.1, p.217-221, Jan. 1992.

BUFFO, R.; REINECCIUS, G.A. Optimization of gum acacia/modified starches/maltodextrin blends for the spray drying of flavours. Perfumer and Flavorist, Wheaton, v.25, n.1, p.37-49, May 2000.

CANO-CHAUCA, M.; STRINGHETA, P.C.; RAMOS, A.M.; CAL-VIDAL, J. Effect of the carriers on the microstructure of mango powder spray drying and its functional characterization, Innovative Food Science \& Emerging Technologies, v.6, p.420-428, 2005.

CHAMBI，H.N.M.; ALVIM，I.D.; BARRERAARELLANO, D.; GROSSO, C.R.F. Solid lipid microparticles containing water-soluble compounds of different molecular mass: Production, characterization and release profiles, Food Research International, v.41, p.229-236, 2008.

COLOMBO, V.E.; GERBER, F. Structures and properties of stabilized vitamin and carotenoid dry powders, Food Structure, v.10, p.161-170, 1991.

EASTMAN, J.E.; MOORE, C.O. Cold water soluble granular starch for gelled food composition. U.S. Patent 4465702, 1984.

COSTA, J.M.G.; BORGES, S.V.; HIJO, A.A.C.T.; SILVA, E.K.; MARQUES, G.R.; CIRILLO, M.Â.; DE AZEVEDO, V.M. Matrix structure selection in the microparticles of essential oil oregano produced by spray dryer, Journal of Microencapsulation, v.30, p.717-727, 2013.

FENNEMA, O.R. Water and Ice. En: Food Chemistry, (Fennema OR Ed.), p. 17-94. Marcel Dekker Inc, New York, 1996.

GHARSALLAOUOI, A.; ROUDAUT, G.; CHAMBIN, O.; VOILLEY, A.; SAUREL, R. Applications of spray-drying in microencapsulation of food ingredients: an overview, Food Research International v.40, p.1107-1121, 2007.

HLA, P.K.; HOGEKAMP, S. Wetting behaviour of 
instanized cocoa beverage powders. International Journal of Food Science and Technology, v.34, p.335-342, 1999.

LIMA, A.; SILVA, A.M.O.; TRINDADE, R.A.; TORRES, R.P.; MANCINI-FILHO, J. Composição química e compostos bioativos presentes na polpa e na amêndoa do pequi (Caryocar brasiliense, Camb.). Revista Brasileira de Fruticultura, v.29, p.695-698, 2007.

MADENE, A.; JACQUOT, M.; SCHER, J. Y DESOBRY, S. Flavour encapsulation and controlled release-a review, International Journal of Food Science and Technology, v.41, p.1-21, 2006.

MAIA， J.G.S.; ANDRADE, E.H.; SILVA, M.H.L. Aroma volatiles of pequi fruit (Caryocar brasiliense, Camb.), Journal Composition and Analysis, v.21, p.574-576, 2008.

MIRANDA-VILELA, A.L.; PEREIRA, L.C.S.; GONÇALVES, C.A.; GRISOLIA, C.K., Pequi fruit (Caryocar brasiliense Camb.) pulp oilreducess exercise-induced inflammatory markers and blood pressure of male and female runners, Nutrition Research, v.29, p.850-858, 2009.

MOYANO, M.J.; MELÉNDEZ-MARTÍNEZ, A.J.; ALBA, J.; HEREDIA, F.J.A., Comprehensive study on the colour of virgin olive oils and its relationship with their chlorophylls and carotenoids indexes (I): CIEXYZ non-uniform colour space, Food Research International, v.41, p.505-512, 2008.

O'BRIEN, R.D. Fats and oils: formulating and processing for applications. Crc Press, London, 2004.
ONEDA, F.Y; RÉ, M.I. The effect of formulation variables on the dissolution and physical properties of spray-dried microspheres containing organic salts, Powder Technology, v.130, p.377-384, 2003.

RODRÍGUEZ-HUEZO, M.E.; PREDROZAISLAS, R.; PRADO-BARRAGAM, L.A.; BERISTAIN, C.I.; VERNON- CARTER, E.J. Microencapsulation by spray drying of multiple emultions containing carotenoids, Journal Food Science, v.69, p.E351- E359, 2004.

SHEU, T.Y.; ROSENBERG, M. Microestructure of microcapsules consisting of whey proteins and carbohydrates, Journal Food Science, v.63, p.491-494, 1998.

SOUSA, A.S.; BORGES, S.V.; MAGALHÃES, N.F.; RICARDO, E.V.; AZEVEDO, A.L.D. Spray dried tomato powder: reconstitution properties and colour. Brazilian Archives of Biology and Technology, v.51, p.807-814, 2008.

TEIXEIRA, M.I.; ANDRADE，L.R.; FARINA, M.; ROCHA-LEÃO, M.H. Characterization of short chain fatty acid microcapsules produced by spray drying. Materials Science and Engineering, v.24, p.653-658, 2004.

TRINDADE, M.A.; GROSSO, C.R.F. The stability of ascorbic acid microencapsulated in granules of rice starch and in gum arabic. Journal of Microencapsulation, v.17, p.169-176, 2000.

TURCHIULI, C.; FUCHS, M.; BOHIN, M.; CUVELIER, M.E.; ORDONNAUD, C.; PEYRATMAILLARD, M.N.; DUMOULIN, E. Oil encapsulation by spray drying and fluidized bed agglomeration. Journal of Food Engineering, v.75, p.27-35, 2006. 